Ein Exemplar dieser sehr eigenthümlichen Art von unbekannter Herkunft in der Sammlung Jules Verreaux's. Die Schnabelform scheint genügend die constante Unterscheidung dieses Vogels zu bezeichnen. Die bei den Zosterops so ungewöhnliche roströthliche Färbung einzelner Theile war wobl geeignet beim ersten Anblick den Eindruck des Unächten hervorzubringen, erwies sich aber bei näherer Untersuchung als natürlich.

\title{
Dunkel bleiben:
}

1. Zosterops obscura Hombr. Jacquin. Voy. au Pol sud Atl. Zool. pl. 20. fig. 6. - Pucher. Text. p. 97. Von unbekannter Herstammung.

2. Zosterops ambigua Sw. Anim. in Menag. p. 295. Cayenne? In der Sammlung Jules Verreaux's befindet sich ein mit? als ambigua Sw. bezeichnetes Exemplar; augenscheinlich ein jüngerer noch nicht ausgefärbter Vogel von höchst unsicherem Gepräge und unbekanntem Habitat.

Reichenbach's Idee, Wilson's Sylvia rubricapilla, eine bekannte nordamericanische Sylvicoline, den Zosterops beizuzählen (Handb. spec. Ornithol. p. 93), verdient nur als Curiosum Erwähnung.

Zosterops? glaucura Reichb. (L'Heorotaire bleu Vieill. Ois. dor. II. pl. 83) bleibt gänzlich dunkel. Mit Recht hebt Reichenbach hervor, dass es sehr irrthümlich sei, diese Abbildung auf $Z$. dorsalis $\mathrm{zu}$ beziehen, wie oft geschehe.

\section{Beobachtungen aus den Jahren 1863 und 1864 in Anhalt.}

\section{Von \\ W. Pässler.}

Wahrscheinlich wird auch in andern Gegenden und Ländern, wie in Anhalt am rechten Elbufer, die Beobachtung gemacht worden sein, dass im späten Frühlinge des Jahres $\mathbf{1 8 6 4}$ die Sommervögel meistens später von ihrer Herbstwanderung zurückkehrten als das Jahr vorher und auch später brüteten. Auch der Herbstzug des Jahres 1864 bot manche Unregelmässigkeiten dar. So bemerkte ich in den ersten Tagen des Septembers noch zwei Nachtigallen, vermuthlich junge, in meinem Garten, und beobachtete den 15. und 16. October grosse Schaaren Schwalben, die lange über meinem Pfarrdorfe kreisten und dann ihre grosse Reise nach 
dem schönen Süden fortsetzten. Da die Hausschwalben schon mit dem August und die Rauchschwalben nach der ersten Woche des Septembers aus nnserer Gegend verschwunden waren, so liegt die Vermuthung nahe, dass jene Spätlinge aus dem hohen Norden gekommen waren. Indess habe ich niemals früher in so weit vorgerückter Jahreszeit eine Schwalbe bemerkt.

Ich will nun Einiges aus meinen Aufzeichnungen der beiden letzten Jahre folgen lassen.

Falco peregrinus behauptet standhaft sein Brutrevier in einem 80-100jährigen Kiefernbestande, obwohl das Pärchen alljährig seiner Eier beraubt wird, zweimal das Männchen und $\mathbf{1 8 6 3}$ das Weibchen beim Ausnehmen des Horstes weggeschossen worden ist. Den 13. oder 14. April ist der Horst gewöhnlich seiner 3 Eier, so namentlich im Jahre 1863 am letztern Tage, beraubt worden. In diesem Jahre dagegen enthielt er erst den 23. April 4 klare Eier. Aber schon Anfangs des Monates belebten die Alten ihr Brutrevier durch lebhaftes und weithin schallendes: „Träht träht."

Falco nisus ward den 24. Mai 1863 in einem Feldholze aut einer Rüster brütend gefunden. Der Horst stand nicht höher als 20 Fuss dicht am Stamm des keineswegs alten und hohen Baumes, war auf einer Unterlage von Reisern mit Moos, Laub und Erde gebaut, mit Reisern, Wurzeln und Haaren ausgelegt und enthielt 4 Eier. Das abgeflogene Weibchen schrie kläglich als der Baum erstiegen und der Horst ausgenommen ward.

Pernis apivorus hat im Jahre $\mathbf{1 8 6 4}$ wieder in einem Feldholze unweit Mühlstedt gehorstet. Ich entdeckte den Horst erst Ende Juni, als er bereits 2 Junge enthielt und hatte später öfter Gelegenheit, die Alten mit den Jungen zu beobachten.

Buteo vulgaris. Horste mit 3 Eiern um die Mitte des März 1863. Im Jahre 1864 erst nach der ersten Woche des April, und zwar stets nur zwei Eier.

Zwei Eier von Otus vulgaris erhielt ich in diesem Jahre Anfangs Mai.

Caprimulgus europaeus schenchte ich den 11. Juni 1864 rom Boden auf. Der Vogel hatte auf einem Ei gesessen, das auf trocknem abgestorbenem Moose lag. Es war weder eingeführtes Gewurzel, noch irgend eine Vertiefung zu bemerken. Da das Nest in einem wenig betretenen Birkenbestande sich befand, liess ich das, vom Vogel erwärmte, Ei liegen. Nach drei Tagen besuchte ich die Brutstelle, um das ganze Gelege, das bekanntlich in nur 
2 Eiern besteht, zu nehmen. Das Nest war leer, und trotz sorgfältiger Durchsuchung des ganzen Reviers auch kein Vogel zu entdecken.

Hirundo riparia hatte im Jahre 1863 schon Anfangs Juni Nester mit der vollen Eierzahl, im Jahre 1864 den 17. Juni noch leere Nester.

Hirundo rustica kam 1863 den 19, den 23. April in diesem Jahre bei uns an, in dem $\mathbf{l}$ Stunde nördlich gelegenen Natho um einige Tage früher.

Hirundo urbica trifft jedes Jahr später ein als die Rauchschwalbe und verlässt uns um eine bis zwei Wochen früher.

Alcedo ispida hatte den 27. August 1864 fünf stark bebrütete Eier in einer, schon mehrfach benutzten, Bruthöhle. Ich habe die sichere Erfahrung, dass Eisvögel in einem Jahre zwei Bruten gemacht haben.

Coracias garrula nistete früher ziemlich häufig in hiesiger Gegend, jetzt in den dazu geeigneten Revieren nur noch in einzelnen Paaren. Ein ziemlich ausgedehntes, mit Eichen, Buchen, Birken, Rüstern und Ellern bestandenes Feldholz wurde früher von vier Pärchen bewohnt; seit sieben Jahren aber gab es keine Mandelkrähen mehr in demselben, obgleich sie früher alljährlich ungestört ihr Brutgeschäft betrieben hatten. In diesem Jahre hatte sich wieder ein Pärchen eingefunden. Ich bemerkte es zuerst den 27. April. Leider wurde einer der Gatten geschossen, worauf der andere das Revier verliess. Im Rosslauer und Spitz. berger Forste dagegen, auch in einzelnen, auf einer Waldwiese stehenden Eichen haben mehre Pärchen genistet. Im Juli bemerkte ich die Alten mit ihren Jungen.

Upupa epops hörte ich $\mathbf{1 8 6 4}$ zuerst den 21. April; hatte in der ersten Woche des Juni ausgeflogene Junge.

Picus martius nistet alljährlich in einem alten Kiefernbestande, welchen die Eisenbahn nach Berlin durchschneidet. Mir war die Bruthöhle des einzigen Pärchens Schwarzspechte, welches unsere schönen Wälder bewohnt, schon seit dem Jahre 1849, wo ich zuerst am 4. April diese Art hörte und sah, bekannt. Sie befindet sich in einer glatten Kiefer, deren Zweige erst über dem kreisrunden Eingange der Bruthöhle ihren Anfang nahmen. Letztere war also für mich unzugänglich, und so sah ich manches Mal wohl einen Jungen den Schnabel und Hals aus dem Loche hervorstrecken, konnte aber dem, ungefähr 60 Fuss über dem Erdboden 
sich befindenden Neste nicht nahen, um die Eier zu erbeuten. Im April des Jahres 1863 ist es aber dem mir befreundeten Revierjäger Behr aus Rosslau gelungen, den Baum zu ersteigen und 5 Eier zu erlangen. $\mathrm{Er}$ hat sich zu diesem Zwecke lange und starke Nägel schmieden lassen, hat, von den obersten Sprossen einer langen Leiter aus anfangend, einen Nagel nach dem andern die Höhe hinauf in den Stamm eingeschlagen, indem er jedesmal auf den eingeschlagenen Nagel sich geschwungen, um in weiterer Höhe eine anderweite Stütze für seinen Fuss zu befestigen, bis er auf dieser luftigen Treppe die Nisthöhle erreicht hat. Um sich vor dem Herunterstürzen zu bewahren, hat der kühne Kletterer ein Seil um sich und den Baum geschlungen, welches ihn in Verbindung mit dem Stamm erhalten haben würde, wenn Hand oder Fuss ihm abgeglitten wäre. Acht Tage darauf sieht Freund Behr den Schwarzspecht in dieselbe Nisthöhle hineinschlüpfen und kommt, da er den Eingang derselben zu erweitern nicht nöthig gehabt hatte, um zu den Eiern zu gelangen, auf den Gedanken, dass das Pärchen zu einer zweiten Brut Anstalt treffen werde. Er hatte sich nicht geirrt, fand aber, als er nach Verlauf von noch nicht ganz 4 Wochen die Bruthöhle untersuchte, kleine Junge in derselben. Es ist also durch diese Thatsache constatirt, dass der Schwarzspecht seine Eier derselben Bruthöhle zum zweiten Male anvertraut, wenn ihm das erste Gelege genommen ist, ohne dass der Eingang der Nisthöhle von dem Eindringling erweitert wurde.

Aus Pommern erhielt ich Eier des Schwarzspechts, welche 1859 den 15,1860 den 5. Mai, 1861 den 25. April, angeblich als klare Eier dem Neste entnommen worden sind. Von

Picus viridis wụrden 1863 fünf Eier den 16. und 1864 den 30. Mai fünf klare Eier gefunden. Von

Picus major 1863 den 8 . Mai, 1864 den 13. und 15. Mai je 5 klare Eier. Von

Picus medius 1863 den 4. Mai 9 Stück, 1864 den 25. Mai 5 Stück. Von

Picus canus empfing ich aus Krain Eier, die im Jahre 1859 angeblich den 28. Mai, und von Picus minor Eier, von denen 5 Stück den 8. Mai gefunden waren.

Cuculus canorus hörte ich $\mathbf{1 8 6 3}$ den 29. April zum ersten Male rufen. Am häufigsten habe ich Kuckukseier in den Nestern der Calamoherpe arundinacea gefunden, sowohl in Rohrteichen als auch in den Weidenwerdern an der Elbe, wo diese Art häufig in Ge- 
sellschaft mit Cal. palustris nistet. Nester des Sumpfrohrsängers habe ich seit dem Jahre 1848 alle Jahre in ziemlicher Anzahl gefunden, aber nur eins mit einem Kuckuksei, das ich noch besitze und das den Eiern der Nesteigenthümer täuschend ähnlich siéht, sich nur durch die Grösse von ihnen unterscheidet. Viele Eier des Kuckuks fand ich auch in den Nestern der S. nisoria und nicht wenige in denen der Motacilla alba, die gern im aufgeklafterten Holze die Wiege ihrer Jungen aufschlägt. Daher sind dergleichen Holzstrecken ergiebige Fundorte von Kuckukseiern. Darum besuche ich alljährlich wiederholt die eine Stunde von meinem Wohnorte entfernte Schlangengrube, eine hart an der Elbe und im Laubwalde gelegene Holzstrecke, auf welcher Tausende von Klaftern Holz aufgestellt sind, in denen viele Pärchen Bachstelzen und Steinschmätzer nisten. Hier machte ich 1863 folgende merkwürdige Erfahrung: Ich fand den 9. Mai ein Kuekuksei im Neste der Mot. alba mit 2 Eiern der Nesteigenthümer. Ich nahm die Eier, sie waren ganz klar, und das Kuckuksei sah gelbgrün aus, eine Färbung, wie sie am öftersten vorkommen. Acht Tage darauf fand ich ein gleiches Kuckuksei in demselben Neste und den 22. Juni ein drittes, und die Vermuthung liegt nahe, dass alle drei Eier von demselben Weibchen herrühren. Jedenfalls wird durch diese Beobachtung die Behauptung umgestossen, die von Mehreren ausgesprochen worden ist, dass ein Kuckuk-Weibchen kein Ei in ein leeres Nest lege.

Im Jahre 1864 hörte ich den Kuckuk zuerst den 9. Mai rufen und fand den 11. Juni 2 Eier in zwei Nestern der S. nisoria ron der gewöhlichen grüngelben Färbung.

Mit aller Entschiedenheit spreche ich mich noch gegen eine andere Behauptung bezüglich der Kuckukseier aus, dass nämlich die grössern Eier, welche man in den Nestern der Sänger finde, wenn sie den Eiern der Nesteigenthümer ähnlich sähen, keine Kuckukseier, sondern Doppeleier der Nestinhaber wären. Es kommen monströse Eier nicht selten von Hausvögeln, zuweilen auch in der Freiheit gelegte vor, wenn auch nicht so häufig als Spar eier; aber jeder Kenner wird sicher sein, ob er ein Kuckuksei oder ein monströses Ei einer Sängerart unter den Händen hat, da die erstern eine viel festere Schale haben, welches dann besonders auffällt, wenn man die zum Entleeren nöthigen Stiche in die verschiedenen Eier macht. Noch überzeugender spricht gegen jene Behauptung Folgendes: Man findet oft Kuckukseier, die den 
Eiern der Nesteigenthümer ganz unähnlich, dagegen den Eiern anderer Sänger sehr ähnlich sehen. So fand ich am I. Juni 1858 ein matt geflecktes grünweisses Ei nebst vier lebhaft gelbroth gefleckten frischen Eiern des Lanius collurio; den 3. Juni ein ganz gleiches stark bebrütetes Kuckuksei neben 3 bebrüteten Eiern der Fring. chloris, welches nach seiner Färbung wohl fur ein Doppelei des Grünlings hätte ausgegeben werden können. So fand ich Eier von der Färbung der Baumpiepereier in Nestern der Cal. arundinacea, die in einem Baumpieperneste gefunden, wohl für monströse Eier des Baumpiepers hätten angesehen werden können. Endlich spricht gegen jene irrige Behauptung ein Umstand, der uns Sammlern oft recht unangenehm ist, dass nämlich die Kuckukseier gar sehr in Sammlungen verbleichen und dadurch oft den Eiern der Nesteigenthümer, denen sie frisch sehr ähnlich sahen, später unähnlich werden. Wie das zuerst gelegte Ei eines Kuckuks aussieht, so sehen alle seine später gelegten Eier von demselben Jahre aus. Das erste Ei wird stets den Eiern der Nestinhaber ähnlich, die folgenden denselben ähnlich oder unähnlich sein, je nachdem sie Nestern derselben Art, wie das erste, oder anderer Sängerarten anvertraut werden.

Während wir nun nicht durch monströse Eier getäuscht zu dem Glauben gekommen sind, dass Kuckukseier die Färbung der Nesteigenthümer-Eier haben; während vielmehr dieser Glaube auf vielfachen sichern Beobachtungen, auf Wahrheit beruht, so ist doch die entgegengesetzte Täuschung möglich: man kann Kuckukseier übersehen und sie für Eier der Nestinhaber halten. Hätte ich z. B. die lerchenartigen Kuckukseier, die ich in den Nestern der Teichrohrsänger fand, in Lerchennestern gefunden, ich wäre nicht darauf gekommen, Kuckukseier vor mir zu sehen, da sie weder durch Grösse noch durch die Färbung sich von Lercheneiern unterscheiden. So können auch Kuckukseier neben Würgereiern liegen, ohne dass man sie erkennt. Ich wenigstens besitze deren, die an Grösse und Färbung den Eiern des rothrückigen Würgers zum Verwechseln ähnlich sind.

Ich besitze ein Ei von der Färbung der Rohrammereier, von letztern nur durch Grösse und etwas festere Schale unterschieden. Ich halte es für ein Kuckuksei und habe dafür folgenden Grund. Ich fand das Nest mit diesem einen Ei. Acht Tage später brütete die Rohrammer auf diesem einen $\mathrm{Ei}$, in welchem sich ein ziemlich entwickeltes $V$ ögelchen fand, das wahrscheinlich wenige Tage später 
ausgeschlüpft wäre. Nun kenne ich kein Beispiel, dass ein Sänger ein einziges Ei ausgebrütet hätte, nachdem ihm die übrigen Eier genommen, es müsste denn dieses eine Ei ein Kuckuksei sein. Nur ein Kuckuksei bebrüten die Vögelchen auch dann noch, wenn ihre eignen Eier zu Grunde gegangen, resp. von dem Kuckuk nach und nach aus dem Neste entfernt worden sind aus Sorge für den eignen Nachkommen. Vielmehr verlassen die Sänger das Nest, wenn man ihnen von dem vollen Gelege ihrer Eier nicht drei Eier lässt. Bis drei können die Vögel zählen, weiter hinauf reicht ihre Rechenkunst nicht. Sie vermissen also das vierte, fünfte, sechste Ei nicht. Das Nest dagegen, aus welchem man die Eier bis auf $\mathrm{zwei}$ entfernt hat, geben sie auf und bauen ein neues Nest für eine andere Brut. Nur bei Parus caudatus habe ich die Erfahrung gemacht, dass man das jedesmal gelegte Ei wegnehmen kann und dass diese kleine Meise jeden Tag wieder ihr Ei in das leere Nest legt. Aus dem oben angeführten Grunde halte ich das bezeichnete Ei für ein Kuckuksei und nicht für das monströse Ei der Rohrammer.

Lanius collurio hatte 1863 schon den 16. Mai Eier. Das Nest stand auf einem Pflaumenbaume meines Gartens. Ich schob dem Würger ein Finkenei und dem Finken ein Würgerei unter. Der Würger hatte das fremde Ei schon nach einer Stunde aus dem Neste entfernt; der Fink duldete es unter seinen Eiern. Leider ward das Finkenpärchen durch einen mir unbekannten Vorfall gestört und verliess das Nest. Ich konnte also nicht beobachten, was es mit dem ausgebrüteten Würger angestellt haben würde.

Muscicapa luctuosa zieht alljährlich durch meinen Garten. Vergeblich habe ich versucht, das Pärchen durch ausgehängte Brutkästen zu fesseln. Es weilte mehrere Tage in meinem und den Nachbargärten, zog dann weiter in den schönen Eichenwald, um mit vielen seines Gleichen zu nisten. Im Jahre 1863 wurden die Vögel in den letzten Tagen des April, 1864 später bebbachtet. Eier findet man gegen das Ende des Mai. Das Weibchen bebrütet die Eier so emsig, dass es leicht auf dem Neste zu fangen ist.

Saxicola oenanthe kommt nach der ersten Woche des April bei uns an. Auch $\mathbf{1 8 6 4}$ bemerkte ich zuerst ein Männchen am 10. April. Sie nisten in unserer Gegend überaus häufig in den aufgerutheten Steinhaufen, und sind rothgefleckte und gestrichelte Eier gar nicht selten.

Pratincola rubetra fand ich den 19. Juni 1863 mit 3 Eiern am 
Rande eines Weidenwerders auf einer Elbwiese. Alle Eier, die ich von dieser Art aus hiesiger Gegend erhalten habe, sehen bleichgrün und ungefleckt. aus, während die aus dem Diebziger und Michelnschen Bruche ein lebhaftes, glänzendes Dunkelgrün, zuweilen mit mattröthlichem Schattenkranze an der Basis zeigen.

Turdus pilaris bemerkte ich noch in den letzten Tagen des April in einem an eine grosse Wiese stossenden Waldestheile, wo ich am 10. Mai 1845 drei Nester mit der vollen Eierzahl gefunden hatte. Ungeachtet der sorgfältigsten Durchforschung dieser und anderer Localitäten, wie die Wachholderdrossel sie zu ihrem Brutgeschäfte liebt, habe ich später weder ein Nest gefunden, noch selbst Vögel bemerkt. In den sechs Stunden von hier entfernten Riegelsdorfer Forsten und in Brandt's Haide sollen sie aber alle Jahre brüten.

Turdus viscivorus, vor 12 Jahren noch so häufig in unsern Kiefernwäldern, ist und bleibt daraus verschwunden. Auch aus den ausgedehnten, fünf Stunden entfernten Wäldern bei Schweinitz hat sich die Misteldrossel, wie mir berichtet worden ist, ganz weggewöhnt.

Sylvia hortensis ist in ebenso grosser Anzahl in unsern Wäldern, welche Unterholz haben und in den Weidenwerdern der Elbe wie die gemeine S. cinerea. Das letzte GartengrasmückenNest fand ich in diesem Jahre den 12. August.

Phyllopneuste rufa, in frühern Jahren in meiner Umgebung nur auf dem Frühjahrszuge gehört, brütete heuer in nicht wenigen Pärchen, wahrscheinlich auch in buschreichen Gärten unfern dem meinigen; wenigstens habe ich dort und hier die Männchen singen hören und sein munteres Treiben belauseht. Vor allen Laubvögeln sind diese Nester am leichtesten zu finden; da sie Schilfbüschel, Eichenbüsche mit trocknem, vorjährigen Laube für ihr Nest lieben und dasselbe auch im Grase nicht so tief verbergen, wie die Gat. tungsverwandten.

Das Nest von Phyll. sibilatrix ist dann am schwersten zu finden, wenn es in unbewachsenen Boden, ins Erdmoos eingebaut ist. Mir flog einst der brütende Vogel vor den Füssen heraus und doch fand ich das Nest nicht. Ein andermal bezeichnete ich zwei gelehrten Herren den Standort des Nestes dadurch, dass ich meinen Hut darüber deckte. Sie vermochten kein Nest zu entdecken und wollten, ärgerlich darüber, dass ich sie zum Besten habe, die Stelle verlassen, worauf ich ihnen das Nest zeigte. Es war in die 
Erde eingebaut, der Eingang lag mit dem Boden in einer Ebene, und eine Erhöhung im Moose gab's nicht. Man musste den Kopf bis an den Boden drücken, um den Eingang zu entdecken.

Phyllopneuste sylvestris. Ich halte die Art fest, obschon sie mir seit Jahren nicht vorgekommen ist. Ich will kein alzugrosses Gewicht auf den Gesang legen', der die Strophen des Fitis- und Weidenlaubvogels vereint. Ich will anch zugestehen, dass Phyll. trochilus zuweilen sehr ähnliche Eier gebe; aber das Nest ist himmelweit verschieden; es bauet überhaupt kein europäischer Vogel ein nur entfernt ähnliches Nest. Es ist so eigenthümlich, dass derjenige es nicht wieder vergisst, wer es nur einmal gesehen hat. Ich habe eins Jahre láng gehabt, bis es zuletzt zerfiel. Es ist in diesen Blättern, sowie in unserm Werke: „Die Eier der Vögel Europas" genau, wenn auch aus dem Gedächtnisse, von mir beschrieben.

In einem Briefe an Herrn Prof. Jäckel frägt Herr Prof. Blasius in Bezug auf meine Angabe, dass ich das Weibchen des MeisnerSängers beim Bauen beobachtet habe, während sich das Männchen singend in den Kronen der nahen Bäume herumgetrieben: „woher wusste Pässler, welches das Männchen und welches das Weibchen war?" Die Antwort auf diese Frage liegt doch gar zu nahe. Es giebt ja ein untrügliches Kennzeichen, die Geschlechter der Vögel, bei denen Männchen und Weibchen ein gleiches Kleid tragen, auch in der Freiheit zu unterscheiden: den Gesang.

Obgleich mir mein bezüglicher Bericht ebenso wenig wie jene Frage des ausgezeichneten Ornithologen vorliegt, so erinnere ich mich doch genau des Factums, wie das Weibchen emsig Nestmaterial zusammentrug und es an dem entstehenden Neste verwendete, dabei ron dem Männchen zuweilen theilnehmend begleitet ward, und wie das Männchen dann munter in den Zweigen der Birken sich umhertreibend seinen Gesang hören liess. Wie oft habe ich Wald- und Fitislaubvögel beim Bau ihrer Nester beobachtet und alsbald gewusst, welches der muntern Thierchen das Männchen, welches das Weibchen war! Letzteres ist ja auch der Baumeister.

Hypolais vulgaris, die alljährlich in meinem Garten brütet, blieb in diesem Frühjahre so lange aus, dass ich schon darauf verzichtete, das liebenswürdige Pärchen, das mich jedes Früh. jahr durch seinen heiteren Gesang, seine Harmlosigkeit und seine Emsigkeit im Aufziehen der Jungen erfreute, in der Nähe zu haben. 
Erst den 27. Maimorgen begrüsste mich der Gesang meines Gartenlaubvogels und an demselben Tage erblickte ich auch das Weibchen, welches schon den 30. auf einen dichtbelaubten Birnbaum zu bauen anfing.

Calamoherpe palustris. Den 30. Mai 1863 Nester mit 3 und 5 Eiern, eins sogar mit 5 bebrüteten Eiern. Im Jahre 1864 den 23. Juni 2 und 3 Eier.

Ebenso hatte $\mathrm{Cal}$. arundinacea den 30. Mai 1863 schon volles Gelege, wie den 23. Juni 1864 noch nicht die volle Eierzahl.

Troglodytes parvulus nistet alljährlich in dem nahen Buchholze. Vier Pärchen bauen theils in die Höhlungen alter moosbedeckter Elsenstämme oder der Bäume, theils unter einer Brücke. Im Jahre $\mathbf{1 8 6 3}$ hatte ich Veranlassung, die sinnreiche Anbringung eines dieser Nester zu bewundern. Der Stamm einer mit Moos überzogenen Rüster war in Manneshöhe gespalten und über der Oeffnung wieder zusammengewachsen. Diese Oeffnung hatten die Vögelchen mit Moos ausgefüllt und in diesem umfangreichen Unterund Ueberbau ihr Nest angebracht. Es enthielt den 7. Mai 6 Eier. Accentor modularis. Ich habe öfter die Nester der zweiten Hecke gefunden als die der ersten. Die Brutzeit der ersten fällt hierorts Anfangs und Mitte April, die der zweiten Anfangs und Mitte Juni. Im Harze baueten die Vögel nur in alte, umfangreiche, mit Moos überzogene Dornbüsche; in hiesiger Gegend fand ich es in kleinen Dornbüschen, einst in einem am Abhange stehenden Busche dicht an der Erde; öfter aber im troknen Schneideschilf, womit die Weidenbüsche der Elbwerder durchwachsen sind. Die Eier unserer Gegend sehen viel blässer aus als die schönen dunkelgriinen, wenn auch glanzlosen, die ich noch aus dem Harze besitze. Auch entwickeln unsere Ebenen-Bewohner keinen so ausgezeichneten Geschmack im Nestbau, wie jene Gebirgsbewohner. Reizend sieht das Nest aus, welches sie aus frischem Moose bauen; noch reizender das Moosnest, welches sie mit rothen, zarten Moosstengeln auskleiden.

Parus cristatus hatte sich einen eigenthümlichen Nistort zurecht gemacht, einen abgestockten, über die Erdfläche nicht emporragenden Pfahl hatte das Pärchen vollends ausgehöhlt und auf den Boden dieses horizontalen, fast $\frac{1}{2}^{\prime}$ tiefen Loches gebaut.

Parus ater traf ich den 10. Mai 1863, seine nackten Jungen deckend. Nest in der Höhle einer Erle, ungefähr zwei Manneslängen über der Erde. 
Parus caudatus den 2. Mai Nest in einem Dornbusche 2' über dem Erdboden mit 10 Eiern. Grosses Vergnügen gewährt es, diese harmlosen Thiere beim Nestbau zu beobachten. Beide Gatten sind dabei gleich emsig, lebhaft und laut, und kümmern sich nicht um den aus nächster Nähe sie beobachtenden Menschen. Selten aber gelingt es, ein vollständiges dieser kunstvollen Nester zu erhalten, da die Vögel die eine Wand an dem Baum nicht zu bekleiden, sondern den Baum als Wand ihres Nestes selbst zu benutzen pflegen und sich begnügen, daselbst die weichen Federn der innern Auskleidung anzubringen.

Nucifraga caryocatactes zeigt sich diesen Herbst in unsern Wäldern. Unsere Forstbeamten sagen: das Erscheinen der Tannenheher bei uns verkündige einen strengen Winter. Andere behaupten das regelmässige Erscheinen dieser Vögel alle sieben Jahre. Man hat Beispiele, dass Pärchen den ganzen Winter über bei uns geblieben sind, ja der Revierjäger Behr hat einst einen Vogel zur Brutzeit bemerkt.

Motacilla sulphurea, die Gebirgsbachstelze, hat mehrere aufeinander folgende Jahre unter einer über die Rossel führenden Brücke genistet. Ich erfuhr es erst im vorigen Jahre, als sie Junge hatten. Dieses Jahr ist das Pärchen nicht erschienen. Im Jahre 1863 fand ich, wo ich nicht irre, den 7. Juli ein Nest mit Jungen im Bodethale unterhalb des Rosstrapp-Felsens, das die Futter zutragenden Alten mir verriethen.

Cynchramus schoeniclus 1863 den 16. Mai mit 6, den 2. Juni mit $\mathbf{5}$ bebrüteten Eiern; $\mathbf{1 8 6 4}$ den $\mathbf{2 3}$. Juni $\mathbf{5}$ bebrütete Eier.

Emberiza hortulana gehört jetzt zu den zahlreichsten Singvögeln der hiesigen Gegend. Wo der Wald an Getreidefeldern grenzt, wo Chausseen und mit Bäumen bepflanzte Feldwege sind, da finden sich auch die Gartenammern. Das Männchen lässt vom Gipfel eines Baumes sein einfaches Lied ertönen, während das Weibchen in einem Hafer-, Gersten- oder Schotenfelde dem Brutgeschäft obliegt. Wegen der gleichmässigen Umgebung ist das Nest schwer aufzufinden, zumal da es nicht gutgeheissen wird, wenn man im Getreide umherwandelt, um nach Nestern zu suchen. Ich habe zuweilen 1 Stunde lang aufgepasst, dass das Männchen seinem Weibchen einen Besuch abstatten oder es im Brüten ablöser möchte. Vergebens! Es pfiff unverdrossen die kurze Strophe seines Liedes, ohne auch nur einmal zur Erde zu fliegen. In frühern Jahren habe ich Eier und Nester aus der Umgegend von Bram- 
bach erhalten, in den letzten Jahren mich aber vergeblich darum bemüht.

Fringilla cannabina in einer Erdhöhle am steilen Ufer der Elbe brütend angetroffen.

Crucirostra curvirostra habe ich Ende Februar in einem Bestande hoher Kiefern die Tannenzapfen verarbeiten sehen. Ich suchte nach Nestern, sah mehrere Vögel, fand aber zu meinem Bedauern kein Nest. Hätte ich einen guten Kletterer Baum für Baum können untersuchen lassen, würde die Mühe vielleicht belohnt worden sein. Früher habe ich nie Kreuzschnäbel in unserer Gegend bemerkt, aber von Jägern gehört, dass sie schon in den hohen Kiefern an der Berliner Eisenbahn genistet hätten.

Columba turtur, die unsere Feldhölzer, Wälder, ausgedehnte, mit dichtem Dorngestrüpp bewachsene Hecken in ziemlicher Anzahl bewohnt, habe ich 1863 den 19. Mai und den 22. Juni brütend angetroffen, die Nester im Dornbusche und in Hopfenranken.

Oedicnemus crepitans häufig auf unsern Brachfeldern, kommt Mitte April, bleibt bis in den October. Nie habe ich einen Vogel auf dem Neste überrascht, nicht einmal von ferne vom Neste wegschlüpfen sehen. In der ersten Hälfte des Mai 2 Eier. Ich fand deren auch im Juni.

Charadrius minor. Hart am östlichen Ausgange meines Pfarrdorfes fliesst die Rossel, deren Bette in Folge der Separation gerade gelegt ist. Früher machte das Flüsschen eine Krümmung an den äussersten Häusern entlang. In diesem alten Bette hat sich Sand aufgehäuft, auf welchem sich im April jedes Frührjahrs ein Pärchen Flussregenpfeifer aufhielt. Der Verkehr ist daneben sehr lebhaft, so dass die Vögel Bedenken finden mussten, zu nisten. Sie waren aber der Menschen so gewohnt, dass sie oft durch die Gassen des Dorfes flogen und die Nähe der Menschen nicht scheueten. An den Kieshägern der Elbe brüten sie in grosser Anzahl. Behr sammelte in einem Jahre ohne Mühe 32 Eier.

Vanellus cristatis mag in diesem Frühjahr mit grossen Nahrungssorgen zu. kämpfen gehabt haben, da nach seiner Ankunft die Gegend nochmals mit ziemlich hohem Schnee bedeckt wurde. In einem nahen Sumpfe nisten alljährlich 4 Pärchen auf den Schilf. kufen, welche schon manches Ei auf unsern Tisch geliefert haben. Die beraubten haben nach 10 Tagen wieder Eier.

Recurvirostra avocetta habe ich im Juni auf den ausgedehnten Elbwiesen unfern Klieken bemerkt. 
Numenius arquatus hielt sich diesen Herbst in grossen Schaaren auf unsern und den Aengern und Wiesen an der Elbe auf. Die Vögel sind ungemein aufmerksam und scheu. Vergebens bemühten sich Gegner, einen zu erlegen.

Ciconia alba nahm seinen Horst auf einer Schener am Ende des Dorfes ein, tödtete aber vor seinem Abzuge die fast flüggen Jungen. Wahrscheinlich ist er derselbe mordlustige Storch, der vor einigen Jahren sein Weib tödtete und sich den andern Tag ein frisches nahm.

Sterna hirundo und minuta wohnen seit einigen Jahren auf einem Kieshäger der Elbe, nahe der Rosslau-Dessauer Brücke. Der Häger hat sich erst seit Jahren gebildet. Bei dem diesjährigen niedern Wasserstande der Elbe wurde es Knaben möglich, hinüber zu waten und die Eier zu zertrümmern. Gleichwohl haben die Vögel, ebenso wie die Flussregenpfeifer, ihr Gebiet behauptet. Die Arten sind daran gewöhnt, dass Menschen und Krähen ihnen die Eier rauben.

Mühlstedt, im November 1864 .

\section{Notizen über den Vogelzug im Herbst 1864, sowie die ornitho- logischen Vorkommnisse in den Ländern der Bischárin, Omáráb and Hadendoa zwischen Berber und Sanakin.}

\section{Von \\ Hofrath Dr. Th. v. Henglin.}

Es war mir gegen Ende meines letzten Aufenthalts am Nil in Berber (August 1864) sehr auffallend, in wenigen Tagen und verhältnissmässig sehr zeitig eine grosse Menge geflügelter Wanderer ans Europa ankommen zu sehen. Ob ausserordentliche meteorologische Erscheinungen im Norden hiermit in Verbindung stehen, kann ich natürlich von hier aus nicht beurtheilen, aber ich glaube, dass die zuerst hier erscheinenden Vorboten des Winters gewöhnlich südlichere Bewohner sind, die auch während der kalten Jahreszeit vielleicht weiter nach Süden vorgehen, als ihre in kältern, nordischen Regionen acclimatisirten Geschwister; denn die ersten Zugvögel, selbst von Arten, die gevöhnt sind in grössern Gesellschaften zu wandern, erscheinen meist Anfangs einzelner.

Am 26. August sah ich am Nil die érsten Upupa. Ardea atricollis und bubulcus brütet um diese Zeit noch. 


\section{$2 \mathrm{BHL}$ Biodiversity Heritage Library}

1865. "Beobachtungen aus den Jahren 1863 und 1864 in Anhalt." Journal $f u$

r Ornithologie 13, 30-42. https://doi.org/10.1007/bf02261151.

View This Item Online: https://www.biodiversitylibrary.org/item/109917

DOI: https://doi.org/10.1007/bf02261151

Permalink: https://www.biodiversitylibrary.org/partpdf/141965

\section{Holding Institution}

Smithsonian Libraries

\section{Sponsored by}

Biodiversity Heritage Library

\section{Copyright \& Reuse}

Copyright Status: Public domain. The BHL considers that this work is no longer under copyright protection.

This document was created from content at the Biodiversity Heritage Library, the world's largest open access digital library for biodiversity literature and archives. Visit BHL at https://www.biodiversitylibrary.org. 